\title{
An Investigation on the Present Situation of Wild Rhododendron Resources and Its Tourism Development and Utilization in Panxi Area
}

\author{
Yuan Ying ${ }^{1}$, Luo Qiang ${ }^{1}$ \\ ${ }^{1}$ (Xichang University College of Agricultural Sciences, Xichang, Sichuan, 615013)
}

\begin{abstract}
Panxi region is an important origin and distribution center of wild Rhododendron spp., which has important ornamental and tourism development value. This study investigated the present situation of wild Rhododendron resources in Sichuan Province, and analyzed the species number, horizontal distribution, vertical distribution, correlation with existing scenic spots and traffic accessibility, and put forward the prospect and suggestions of making full use of the rich wild Rhododendron resources in Panxi area for tourism development and boosting rural revitalization.
\end{abstract}

\section{Introduction}

Rhododendron spp. is a large genus with the most species and characteristics in Ericaceae. There are about 967 species in the world, including 8 subgenera ${ }^{[1]}$ and about 570 species in China, belonging to 6 subgenera, 10 groups and 49 subgroups (Fang Mingyuan et al., 2005), which are distributed everywhere except Xinjiang and Ningxia. Because of its cold resistance, it is mainly distributed in the middle and high mountain areas of northwest Yunnan, southeast Tibet and southwest Sichuan. Rhododendron has important economic value. ${ }^{[2]}$ Rhododendron is one of the world's four largest alpine flowers and one of China's ten famous flowers, with high ornamental value and garden application value. Rhododendron contains ketone chemicals and volatile oil, which has antibacterial, antiinflammatory, antitussive, antiasthmatic and expectorant effects. It can treat many diseases and it has very important medicinal value. Rhododendron plants mostly grow on dry ridges, hilltops or cliffs, which are barren and droughtresistant, with dense branches and developed roots, and often clump into impenetrable shrubbery, which can maintain water and soil and have high ecological utilization value.

Panxi region is located in the southwest of Sichuan Province, on the eastern edge of Hengduan Mountains, and consists of Panzhihua City and Liangshan Prefecture, which are composed of 3 districts, 2 cities (county-level cities) and 17 counties. This area is the core area of the resource "Golden Triangle" in Southwest China, which is known as "plant kingdom" and "biological gene bank". Panxi region takes subtropical climate as the base band and south subtropical climate as the main one, with abundant sunshine, abundant rainfall, distinct dry and wet seasons, small annual temperature difference and large daily temperature difference. In winter, it is sunny and dry due to the sinking of the south branch of westerly wind. In summer, it is rainy and humid under the influence of southwest monsoon. The area is rich in heat, with annual sunshine hours of $2300 \sim 2800 \mathrm{~h}$, annual total solar radiation of $580 \sim 630 \mathrm{KJ} / \mathrm{cm}$ and frost-free period of over 300d. The annual average temperature is $17.5 \sim 21.0^{\circ} \mathrm{C}$, and the temperature difference between day and night is $13.1 \sim 14.4^{\circ} \mathrm{C}$. The annual rainfall is $800 \sim 1200 \mathrm{~mm}$. There are high mountains and deep valleys in Panxi area, and the relative height difference of canyons is about 3000 $\mathrm{m}$. The special geographical environment of "one mountain is divided into four seasons, ten miles are different days" and the unique conditions of "sunshine in the north of China, temperature in the south and climate in the Indian Ocean" have bred abundant wild Rhododendron germplasm resources in Panxi area, making Panxi area an important origin and distribution center of wild Rhododendron plants.

\section{Research methods}

\subsection{Historical data collation}

The Chinese flora, illustrations of Chinese higher plants, papers related to the species and distribution of Rhododendron resources in Panxi area were consulted, and the historical distribution data of Rhododendron plants in Panxi area were obtained and summarized through Chinese virtual herbarium (http://www.cvh.ac.cn/).

\subsection{Field survey statistics}

From April 2016 to June 2020, the project team conducted a field investigation on the species and distribution of wild 
Rhododendron in Panxi area, collected samples and registered the species identification, and analyzed the floristic geographical distribution and ecological causes of wild Rhododendron in Panxi area.

\section{Results and analysis}

\begin{tabular}{|c|c|c|c|c|c|c|}
\hline $\begin{array}{c}\text { Serial } \\
\text { Number }\end{array}$ & Chinese Name & Latin Name & Character & Distribute Counties (Cities) & $\begin{array}{l}\text { Distribution } \\
\text { Altitude }\end{array}$ & Habitat \\
\hline 1 & $\begin{array}{l}\text { Liang Mao } \\
\text { Dujuan }\end{array}$ & $\begin{array}{c}\text { Rhododendron } \\
\text { microphyton Franch. }\end{array}$ & $\begin{array}{l}\text { Evergreen erect } \\
\text { shrub }\end{array}$ & $\begin{array}{l}\text { Xichang, Dechang, Puge, } \\
\text { Huili, Huidong, Miyi, } \\
\text { Mianning and other counties } \\
\text { (cities) }\end{array}$ & $1300-3200 \mathrm{~m}$ & $\begin{array}{l}\text { In the } \\
\text { thicket }\end{array}$ \\
\hline 2 & $\begin{array}{l}\text { Rhododendron } \\
\text { scabra }\end{array}$ & $\begin{array}{c}\text { Rhododendron } \\
\text { scabrifolium Franch. }\end{array}$ & Bush & $\begin{array}{c}\text { Xichang, Mianning, } \\
\text { Yanyuan, Leibo, Yanbian, } \\
\text { Miyi and other counties } \\
\text { (cities) }\end{array}$ & $2200-4000 \mathrm{~m}$ & $\begin{array}{l}\text { Under } \\
\text { forests or } \\
\text { shrubs }\end{array}$ \\
\hline 3 & $\begin{array}{c}\text { Rhododendron } \\
\text { longistamens }\end{array}$ & $\begin{array}{c}\text { Rhododendron } \\
\text { stamineum Franch. }\end{array}$ & $\begin{array}{l}\text { Evergreen shrubs } \\
\text { or small trees }\end{array}$ & Mei Gu, ningnan county & $2400-3100 \mathrm{~m}$ & $\begin{array}{l}\text { Under } \\
\text { forests or } \\
\text { shrubs }\end{array}$ \\
\hline 4 & $\begin{array}{l}\text { Burst stick } \\
\text { flower }\end{array}$ & $\begin{array}{c}\text { Rhododendron } \\
\text { spinuliferum Franch. }\end{array}$ & Bush & Liangshan counties (cities) & $1900-2500 \mathrm{~m}$ & $\begin{array}{l}\text { Under } \\
\text { forests or } \\
\text { shrubs }\end{array}$ \\
\hline 5 & $\begin{array}{l}\text { Rhododendron } \\
\text { pilosa }\end{array}$ & $\begin{array}{c}\text { Rhododendron } \\
\text { pubescens Balf.f. et } \\
\text { Forrest }\end{array}$ & Undershrub & $\begin{array}{l}\text { Xichang, Miyi, Yanbian, } \\
\text { Mianning, Dechang, } \\
\text { Yanyuan, Muli and other } \\
\text { counties (cities) }\end{array}$ & $2700-3500 \mathrm{~m}$ & $\begin{array}{l}\text { In the } \\
\text { thicket }\end{array}$ \\
\hline 6 & $\begin{array}{l}\text { Rhododendron } \\
\text { cosmetology }\end{array}$ & $\begin{array}{c}\text { Rhododendron } \\
\text { calophytum Franch }\end{array}$ & $\begin{array}{l}\text { Evergreen shrubs } \\
\text { or small trees }\end{array}$ & $\begin{array}{c}\text { Counties (cities) in Panxi } \\
\text { area }\end{array}$ & $1300-4000 \mathrm{~m}$ & $\begin{array}{l}\text { Woodlan } \\
\text { d retreat }\end{array}$ \\
\hline 7 & $\begin{array}{l}\text { Rhododendron } \\
\text { axillaris }\end{array}$ & $\begin{array}{l}\text { Rhododendron } \\
\text { racemosum Franch. }\end{array}$ & Undershrub & $\begin{array}{l}\text { Xichang, Leibo, Meigu, } \\
\text { Butuo, Zhaojue, Puge, } \\
\text { Dechang, Yanyuan, Muli, } \\
\text { Miyi, Yanbian and Huili } \\
\text { counties (cities) }\end{array}$ & $1500-3800 \mathrm{~m}$ & $\begin{array}{l}\text { Under } \\
\text { forests or } \\
\text { shrubs }\end{array}$ \\
\hline 8 & $\begin{array}{l}\text { Rhododendron } \\
\text { hybridum }\end{array}$ & $\begin{array}{l}\text { Rhododendron } \\
\text { oreotrephes W. W. Sm. }\end{array}$ & Evergreen shrubs & $\begin{array}{l}\text { Leibo, Butuo, Yanyuan, } \\
\text { Muli, Miyi, Yanbian, Huili } \\
\text { and other counties }\end{array}$ & $1800-3700 \mathrm{~m}$ & $\begin{array}{l}\text { Under } \\
\text { forests or } \\
\text { shrubs }\end{array}$ \\
\hline 9 & $\begin{array}{l}\text { Rhododendron } \\
\text { dewdrop }\end{array}$ & $\begin{array}{l}\text { Rhododendron } \\
\text { irroratum Franch. }\end{array}$ & $\begin{array}{l}\text { Shrubs or small } \\
\text { trees }\end{array}$ & $\begin{array}{l}\text { Miyi, Yanbian County and } \\
\text { Liangshan counties (cities) }\end{array}$ & $1700-3200 \mathrm{~m}$ & $\begin{array}{c}\text { Under } \\
\text { forests or } \\
\text { shrubs }\end{array}$ \\
\hline 10 & $\begin{array}{l}\text { Rhododendron } \\
\text { magna }\end{array}$ & Rhododendron rex Levl. & $\begin{array}{l}\text { Small evergreen } \\
\text { trees }\end{array}$ & $\begin{array}{l}\text { Yanbian, Miyi County and } \\
\text { Liangshan counties (cities) }\end{array}$ & $2300-3300 \mathrm{~m}$ & $\begin{array}{l}\text { Woodlan } \\
\text { d retreat }\end{array}$ \\
\hline 11 & $\begin{array}{c}\text { Rhododendron } \\
\text { dauricum }\end{array}$ & $\begin{array}{c}\text { Rhododendron decorum } \\
\text { Franch. }\end{array}$ & $\begin{array}{l}\text { Evergreen shrubs } \\
\text { or small trees }\end{array}$ & $\begin{array}{c}\text { Counties (cities, districts) in } \\
\text { Panxi area }\end{array}$ & $1000-3300 \mathrm{~m}$ & $\begin{array}{l}\text { Under } \\
\text { forests or } \\
\text { shrubs }\end{array}$ \\
\hline 12 & $\begin{array}{l}\text { Rhododendron } \\
\text { purpurea }\end{array}$ & $\begin{array}{l}\text { Rhododendron amesiae } \\
\text { Rehd. et Wils. }\end{array}$ & Bush & $\begin{array}{l}\text { Jinyang, Ganluo, Mianning, } \\
\text { Muli and other counties }\end{array}$ & $2200-3000 \mathrm{~m}$ & $\begin{array}{l}\text { Woodlan } \\
\text { d retreat }\end{array}$ \\
\hline 13 & $\begin{array}{l}\text { Rhododendron } \\
\text { auriculatum }\end{array}$ & $\begin{array}{c}\text { Rhododendron } \\
\text { auriculatum Hemsl. }\end{array}$ & $\begin{array}{l}\text { Evergreen shrubs } \\
\text { or small trees }\end{array}$ & shaojue county & $1500-2000 \mathrm{~m}$ & $\begin{array}{l}\text { Woodlan } \\
\text { d retreat }\end{array}$ \\
\hline 14 & $\begin{array}{l}\text { Rhododendron } \\
\text { glabra }\end{array}$ & $\begin{array}{c}\text { Rhododendron } \\
\text { vernicosum Franch. }\end{array}$ & $\begin{array}{l}\text { Evergreen shrubs } \\
\text { or small trees }\end{array}$ & $\begin{array}{l}\text { Xichang, Ganluo, Muli, } \\
\text { Yanyuan and Zhaojue } \\
\text { counties (cities) }\end{array}$ & $2650-4300 \mathrm{~m}$ & $\begin{array}{l}\text { Woodlan } \\
\text { d retreat }\end{array}$ \\
\hline 15 & $\begin{array}{l}\text { Rhododendron } \\
\text { tomentosa }\end{array}$ & $\begin{array}{l}\text { Rhododendron } \\
\text { radendum Fang }\end{array}$ & Evergreen shrub & ningnan county & $3000-4100 \mathrm{~m}$ & $\begin{array}{l}\text { Under } \\
\text { forests or } \\
\text { shrubs }\end{array}$ \\
\hline
\end{tabular}

According to the data and investigation results, there are 64 species, 5 varieties and 1 subspecies ${ }^{[5][6][7][8][9]}$ of wild Rhododendron in Panxi area. See Table 1 for the species,

characters and distribution of wild Rhododendron.

\subsection{Species composition}




\begin{tabular}{|c|c|c|c|c|c|c|}
\hline 16 & cuckoo & $\begin{array}{c}\text { Rhododendron simsii } \\
\text { Planch. }\end{array}$ & Sheepberry & $\begin{array}{l}\text { Xichang, Huili, Ningnan, } \\
\text { Leibo, Meigu and other } \\
\text { counties (cities) }\end{array}$ & $1000-2700 \mathrm{~m}$ & $\begin{array}{l}\text { Under } \\
\text { forests or } \\
\text { shrubs }\end{array}$ \\
\hline 17 & $\begin{array}{l}\text { Rhododendron } \\
\text { glabra }\end{array}$ & $\begin{array}{c}\text { Rhododendron nitidulum } \\
\text { Rehd. et Wils. }\end{array}$ & Evergreen shrub & puge county & $2800-3000 \mathrm{~m}$ & $\begin{array}{l}\text { On the } \\
\text { meadow }\end{array}$ \\
\hline 18 & $\begin{array}{l}\text { Rhododendron } \\
\text { multicolour } \\
\text { (Rhododendron } \\
\text { multicolour var.) }\end{array}$ & $\begin{array}{l}\text { Rhododendron rupicola } \\
W . W . \text { Smith var. } \\
\text { muliense (Balf.f. et } \\
\text { Forrest) Philip. et } M . N . \\
\text { Philip. } \\
\end{array}$ & Evergreen shrub & Muli and Yanyuan counties & $3000-4500 \mathrm{~m}$ & $\begin{array}{l}\text { In the } \\
\text { thicket }\end{array}$ \\
\hline 19 & $\begin{array}{l}\text { Rhododendron } \\
\text { multicolor }\end{array}$ & $\begin{array}{c}\text { Rhododendron rupicola } \\
\text { W.W. Smith }\end{array}$ & Evergreen shrub & Muli and Yanyuan counties & $3000-4500 \mathrm{~m}$ & $\begin{array}{l}\text { In the } \\
\text { thicket }\end{array}$ \\
\hline 20 & $\begin{array}{l}\text { Mahonia } \\
\text { fimbristipula }\end{array}$ & $\begin{array}{c}\text { Rhododendron } \\
\text { leptothrium Balf.f.et } \\
\text { Forrest }\end{array}$ & $\begin{array}{l}\text { Shrubs or small } \\
\text { trees }\end{array}$ & ningnan county & $1700-3200 \mathrm{~m}$ & $\begin{array}{l}\text { In the } \\
\text { thicket }\end{array}$ \\
\hline 21 & $\begin{array}{l}\text { Rhododendron } \\
\text { nobilis } \\
\text { (subspecies) }\end{array}$ & $\begin{array}{l}\text { Rhododendron decorum } \\
\text { subsp. diaprepes (Balf.f. } \\
\& W . W . \text { Sm.) T. L. Ming }\end{array}$ & $\begin{array}{l}\text { Green shrubs or } \\
\text { small trees }\end{array}$ & $\begin{array}{c}\text { Counties (cities) in Panxi } \\
\text { area }\end{array}$ & $1000-3300 \mathrm{~m}$ & $\begin{array}{l}\text { Under } \\
\text { forests or } \\
\text { shrubs }\end{array}$ \\
\hline 22 & $\begin{array}{l}\text { Rhododendron } \\
\text { Pink } \\
\text { (Rhododendron } \\
\text { montanum var.) }\end{array}$ & $\begin{array}{l}\text { Rhododendron oreodoxa } \\
\text { Franch. var. fargesii } \\
\text { (Franch.) Chamb. ex } \\
\text { Cullen et Chamb. }\end{array}$ & $\begin{array}{l}\text { Often Green } \\
\text { shrubs or small } \\
\text { trees }\end{array}$ & Butuo county & $1800-3500 \mathrm{~m}$ & $\begin{array}{l}\text { Under } \\
\text { forests or } \\
\text { shrubs }\end{array}$ \\
\hline 23 & $\begin{array}{l}\text { Rhododendron } \\
\text { cinereus }\end{array}$ & $\begin{array}{c}\text { Rhododendron } \\
\text { hippophaeoides Balf.f. } \\
\text { et W. W. Smith }\end{array}$ & Evergreen shrub & $\begin{array}{l}\text { Mianning, Ganluo, Yuexi and } \\
\text { Xide counties }\end{array}$ & $2000-2300 \mathrm{~m}$ & $\begin{array}{l}\text { Under } \\
\text { forests or } \\
\text { shrubs }\end{array}$ \\
\hline 24 & $\begin{array}{l}\text { Rhododendron } \\
\text { tonkinensis }\end{array}$ & $\begin{array}{c}\text { Rhododendron } \\
\text { hypoglaucum Hemsl. }\end{array}$ & Evergreen shrub & meigu county & $1500-2100 \mathrm{~m}$ & $\begin{array}{l}\text { Woodlan } \\
\text { d retreat }\end{array}$ \\
\hline 25 & $\begin{array}{l}\text { Rhododendron } \\
\text { maculatum }\end{array}$ & $\begin{array}{l}\text { Rhododendron } \\
\text { clementinae Forrest }\end{array}$ & Evergreen shrubs & $\begin{array}{l}\text { Jinyang, Meigu and Leibo } \\
\text { counties }\end{array}$ & $3200-4100 \mathrm{~m}$ & $\begin{array}{l}\text { In the } \\
\text { thicket }\end{array}$ \\
\hline 26 & $\begin{array}{l}\text { Rhododendron } \\
\text { concave }\end{array}$ & $\begin{array}{c}\text { Rhododendron } \\
\text { davidsonianum Rehd. et } \\
\text { Wils }\end{array}$ & Bush & $\begin{array}{l}\text { Xichang, Dechang, Leibo, } \\
\text { Muli, Yanyuan, Huili and } \\
\text { other counties (cities) }\end{array}$ & $1500-2800 \mathrm{~m}$ & $\begin{array}{l}\text { In the } \\
\text { thicket }\end{array}$ \\
\hline 27 & $\begin{array}{l}\text { Rhododendron } \\
\text { chrysanthum }\end{array}$ & $\begin{array}{c}\text { Rhododendron lutescens } \\
\text { Franch. }\end{array}$ & Bush & $\begin{array}{l}\text { Leibo, Meigu, Miyi and other } \\
\text { counties }\end{array}$ & $1700-2100 \mathrm{~m}$ & $\begin{array}{c}\text { Under } \\
\text { forests or } \\
\text { shrubs }\end{array}$ \\
\hline 28 & $\begin{array}{l}\text { Rhododendron } \\
\text { xiuya }\end{array}$ & $\begin{array}{l}\text { Rhododendron } \\
\text { concinnum Hemsl. }\end{array}$ & Bush & $\begin{array}{l}\text { Xichang, Leibo, Meigu, } \\
\text { Yuexi, Mianning, Muli, } \\
\text { Yanyuan and other counties } \\
\text { (cities) }\end{array}$ & $2300-3000 \mathrm{~m}$ & $\begin{array}{l}\text { In the } \\
\text { thicket }\end{array}$ \\
\hline 29 & $\begin{array}{l}\text { Rhododendron } \\
\text { truncatum }\end{array}$ & $\begin{array}{l}\text { Rhododendron } \\
\text { orbiculare Decne. }\end{array}$ & $\begin{array}{l}\text { Evergreen shrubs, } \\
\text { rare small trees }\end{array}$ & $\begin{array}{l}\text { Mianning, Meigu and other } \\
\text { counties }\end{array}$ & $2000-3500 \mathrm{~m}$ & $\begin{array}{l}\text { Woodlan } \\
\text { d retreat }\end{array}$ \\
\hline 30 & $\begin{array}{l}\text { Huang Mao } \\
\text { Dujuan }\end{array}$ & $\begin{array}{c}\text { Rhododendron rufum } \\
\text { Batalin }\end{array}$ & $\begin{array}{l}\text { Often Green } \\
\text { shrubs or small } \\
\text { trees }\end{array}$ & $\begin{array}{l}\text { Yuexi, Huidong and other } \\
\text { counties }\end{array}$ & $2300-3800 \mathrm{~m}$ & $\begin{array}{l}\text { Woodlan } \\
\text { d retreat }\end{array}$ \\
\hline 31 & $\begin{array}{l}\text { Rhododendron } \\
\text { Quercus }\end{array}$ & $\begin{array}{c}\text { Rhododendron } \\
\text { phaeochrysum Balf.f. et } \\
\text { W.W. Smith }\end{array}$ & Evergreen shrubs & $\begin{array}{l}\text { Xichang, Muli, Yanyuan, } \\
\text { Huili and other counties } \\
\text { (cities) }\end{array}$ & $3300-4200 \mathrm{~m}$ & $\begin{array}{l}\text { In the } \\
\text { thicket }\end{array}$ \\
\hline 32 & $\begin{array}{l}\text { Rhododendron } \\
\text { dauricum }\end{array}$ & $\begin{array}{l}\text { Rhododendron } \\
\text { thymifolium Maxim. }\end{array}$ & $\begin{array}{l}\text { Evergreen erect } \\
\text { subshrub; } \\
\text { evergreen erect } \\
\text { subshrub }\end{array}$ & $\begin{array}{l}\text { Xichang, Muli, Zhaojue and } \\
\text { other counties (cities) }\end{array}$ & $2400-4800 \mathrm{~m}$ & $\begin{array}{l}\text { Under } \\
\text { forests or } \\
\text { shrubs }\end{array}$ \\
\hline 33 & $\begin{array}{l}\text { Rhododendron } \\
\text { hirsutum }\end{array}$ & $\begin{array}{c}\text { Rhododendron } \\
\text { cephalanthum Franch. }\end{array}$ & Evergreen shrub & Muli county & $3000-4000 \mathrm{~m}$ & $\begin{array}{l}\text { On the } \\
\text { meadow }\end{array}$ \\
\hline 34 & $\begin{array}{l}\text { Rhododendron } \\
\text { Longshu }\end{array}$ & $\begin{array}{c}\text { Rhododendron } \\
\text { przewalskii Maxim. }\end{array}$ & Evergreen shrubs & Muli and Yanyuan counties & $2900-4300 \mathrm{~m}$ & $\begin{array}{l}\text { Woodlan } \\
\text { d retreat }\end{array}$ \\
\hline 35 & $\begin{array}{l}\text { Rhododendron } \\
\text { yunnanensis }\end{array}$ & $\begin{array}{c}\text { Rhododendron } \\
\text { yunnanense Franch. }\end{array}$ & $\begin{array}{l}\text { Deciduous, semi- } \\
\text { deciduous or } \\
\text { evergreen shrubs, } \\
\text { occasionally small } \\
\text { trees }\end{array}$ & $\begin{array}{l}\text { Xichang, Yanyuan, Huili and } \\
\text { other counties (cities) }\end{array}$ & $2000-4000 \mathrm{~m}$ & $\begin{array}{l}\text { Under } \\
\text { forests or } \\
\text { shrubs }\end{array}$ \\
\hline 36 & $\begin{array}{l}\text { Rhododendron } \\
\text { primrose }\end{array}$ & $\begin{array}{l}\text { Rhododendron } \\
\text { primuliflorum Bur. et } \\
\text { Franch. }\end{array}$ & Evergreen shrub & Muli and Yanyuan counties & $3700-4100 \mathrm{~m}$ & $\begin{array}{l}\text { In the } \\
\text { thicket }\end{array}$ \\
\hline
\end{tabular}




\begin{tabular}{|c|c|c|c|c|c|c|}
\hline 37 & $\begin{array}{l}\text { Rhododendron } \\
\text { flavum }\end{array}$ & $\begin{array}{c}\text { Rhododendron wardii } W . \\
W . \text { Smith }\end{array}$ & Bush & Muli and Yanyuan counties & $3000-4000 \mathrm{~m}$ & $\begin{array}{c}\text { Under } \\
\text { forests or } \\
\text { shrubs }\end{array}$ \\
\hline 38 & red azalea & $\begin{array}{c}\text { Rhododendron } \\
\text { neriiflorum Franch. }\end{array}$ & Evergreen shrubs & $\begin{array}{l}\text { Huidong, Huili and ningnan } \\
\text { county }\end{array}$ & $2500-3600 \mathrm{~m}$ & $\begin{array}{l}\text { Woodlan } \\
\text { d retreat }\end{array}$ \\
\hline 39 & $\begin{array}{l}\text { Rhododendron } \\
\text { obscureum }\end{array}$ & $\begin{array}{c}\text { Rhododendron } \\
\text { amundsenianum Hand.- } \\
\text { Mazz }\end{array}$ & Evergreen shrubs & Muli and Yanyuan counties & $3900-4250 \mathrm{~m}$ & $\begin{array}{l}\text { On the } \\
\text { meadow }\end{array}$ \\
\hline 40 & $\begin{array}{l}\text { Rhododendron } \\
\text { Miyi }\end{array}$ & $\begin{array}{c}\text { Rhododendron miyiense } \\
W . K . H u\end{array}$ & Bush & $\begin{array}{l}\text { Miyi, Yanbian, Huili and } \\
\text { Huidong counties }\end{array}$ & $1700 \mathrm{~m}$ & $\begin{array}{l}\text { Woodlan } \\
\text { d retreat }\end{array}$ \\
\hline 41 & $\begin{array}{l}\text { Rhododendron } \\
\text { Xichang } \\
\end{array}$ & $\begin{array}{c}\text { Rhododendron } \\
\text { xichangense Z.J.Zhao } \\
\end{array}$ & Bush & Xichang City & $2400-2600 \mathrm{~m}$ & $\begin{array}{l}\text { Woodlan } \\
\text { d retreat }\end{array}$ \\
\hline 42 & $\begin{array}{l}\text { Rhododendron } \\
\text { rusticus }\end{array}$ & $\begin{array}{l}\text { Rhododendron } \\
\text { siderophyllum Franch. }\end{array}$ & Bush & $\begin{array}{l}\text { Xichang, Yanyuan, Huili and } \\
\text { other counties (cities) }\end{array}$ & $2700-3100 \mathrm{~m}$ & $\begin{array}{l}\text { Under } \\
\text { forests or } \\
\text { shrubs }\end{array}$ \\
\hline 43 & $\begin{array}{l}\text { Rhododendron } \\
\text { rubrum }\end{array}$ & $\begin{array}{l}\text { Rhododendron } \\
\text { rubiginosum Franch. }\end{array}$ & $\begin{array}{c}\text { Often Green } \\
\text { shrubs or small } \\
\text { trees }\end{array}$ & $\begin{array}{l}\text { Xichang, Yanyuan, Huili and } \\
\text { other counties (cities) }\end{array}$ & $2900-3300 \mathrm{~m}$ & $\begin{array}{l}\text { Woodlan } \\
\text { d retreat }\end{array}$ \\
\hline 44 & $\begin{array}{l}\text { Rhododendron } \\
\text { polytrichum }\end{array}$ & $\begin{array}{l}\text { Rhododendron polylepis } \\
\text { Franch. }\end{array}$ & $\begin{array}{l}\text { Shrubs or small } \\
\text { trees }\end{array}$ & $\begin{array}{l}\text { Xichang, Yanyuan and other } \\
\text { counties (cities) }\end{array}$ & $2700-3100 \mathrm{~m}$ & $\begin{array}{l}\text { Woodlan } \\
\text { d retreat }\end{array}$ \\
\hline 45 & $\begin{array}{l}\text { Rhododendron } \\
\text { polytrichum }\end{array}$ & $\begin{array}{l}\text { Rhododendron } \\
\text { augustinii Hemsl. }\end{array}$ & Evergreen shrubs & $\begin{array}{l}\text { Xichang, Yanyuan, Huili, } \\
\text { Zhaojue, Jinyang, Meigu and } \\
\text { other counties (cities) }\end{array}$ & $2300-2700 \mathrm{~m}$ & $\begin{array}{l}\text { Woodlan } \\
\text { d retreat }\end{array}$ \\
\hline 46 & Ki Mao Dujuan & $\begin{array}{l}\text { Rhododendron rigidum } \\
\text { Franch. }\end{array}$ & Bush & $\begin{array}{l}\text { Xichang, Yanyuan and other } \\
\text { counties (cities) }\end{array}$ & $2400-2800 \mathrm{~m}$ & $\begin{array}{l}\text { In the } \\
\text { thicket }\end{array}$ \\
\hline 47 & $\begin{array}{l}\text { Rhododendron } \\
\text { sclerophyllum }\end{array}$ & $\begin{array}{l}\text { Rhododendron } \\
\text { tatsienense Franch. }\end{array}$ & Bush & $\begin{array}{l}\text { Xichang, Yanyuan, Huili and } \\
\text { other counties (cities) }\end{array}$ & $3100-3300 \mathrm{~m}$ & $\begin{array}{l}\text { Woodlan } \\
\text { d retreat }\end{array}$ \\
\hline 48 & $\begin{array}{l}\text { Dianhong Mao } \\
\text { Dujuan }\end{array}$ & $\begin{array}{c}\text { Rhododendron } \\
\text { rufohirtum Hand.-Mazz. }\end{array}$ & Bush & $\begin{array}{l}\text { Xichang, Yanyuan, Huili and } \\
\text { other counties (cities) }\end{array}$ & $1700-2400 \mathrm{~m}$ & $\begin{array}{l}\text { In the } \\
\text { thicket }\end{array}$ \\
\hline 49 & $\begin{array}{l}\text { Rhododendron } \\
\text { floribundum }\end{array}$ & $\begin{array}{l}\text { Rhododendron } \\
\text { floribundum Franch. }\end{array}$ & $\begin{array}{l}\text { Shrubs or small } \\
\text { trees }\end{array}$ & $\begin{array}{l}\text { Xichang, Yanyuan, Huili and } \\
\text { other counties (cities) }\end{array}$ & $2700-3000 \mathrm{~m}$ & $\begin{array}{l}\text { In the } \\
\text { thicket }\end{array}$ \\
\hline 50 & $\begin{array}{c}\text { Rhododendron } \\
\text { crispate } \\
\text { (Rhododendron } \\
\text { variegatum) } \\
\end{array}$ & $\begin{array}{l}\text { Rhododendron } \\
\text { denudatum Levl }\end{array}$ & $\begin{array}{l}\text { Shrubs or small } \\
\text { trees }\end{array}$ & $\begin{array}{l}\text { Xichang, Yanyuan, Huili and } \\
\text { other counties (cities) }\end{array}$ & $2500-3000 \mathrm{~m}$ & $\begin{array}{l}\text { In the } \\
\text { thicket }\end{array}$ \\
\hline 51 & $\begin{array}{l}\text { Broken rice } \\
\text { flower }\end{array}$ & $\begin{array}{l}\text { Rhododendron } \\
\text { spiciferum Franch. }\end{array}$ & Undershrub & $\begin{array}{l}\text { Xichang, Yanyuan, Huili and } \\
\text { other counties (cities) }\end{array}$ & $1800-2700 \mathrm{~m}$ & $\begin{array}{l}\text { Woodlan } \\
\text { d retreat }\end{array}$ \\
\hline 52 & $\begin{array}{l}\text { Rhododendron } \\
\text { purpureum } \\
\text { (Rhododendron } \\
\text { barbadense var.) }\end{array}$ & $\begin{array}{c}\text { Rhododendron } \\
\text { strigillosum Franch. var. } \\
\text { monosematum (Hutch.) } \\
\text { T. L. Ming }\end{array}$ & $\begin{array}{l}\text { Often Green } \\
\text { shrubs or small } \\
\text { trees }\end{array}$ & Xichang City & $2900-3500 \mathrm{~m}$ & $\begin{array}{l}\text { Woodlan } \\
\text { d retreat }\end{array}$ \\
\hline 53 & $\begin{array}{l}\text { Rhododendron } \\
\text { pulveratum }\end{array}$ & $\begin{array}{c}\text { Rhododendron } \\
\text { impeditum Balf.f. et W. } \\
\text { W. Smith }\end{array}$ & Evergreen shrubs & $\begin{array}{l}\text { Xichang City and Yanyuan } \\
\text { County }\end{array}$ & $2500-3100 \mathrm{~m}$ & $\begin{array}{l}\text { On the } \\
\text { meadow }\end{array}$ \\
\hline 54 & $\begin{array}{l}\text { Rhododendron } \\
\text { pseudogalactica } \\
\text { (Rhododendron } \\
\text { magna var.) }\end{array}$ & $\begin{array}{l}\text { Rhododendron rex } \\
\text { Levl.subsp.fictolacteum( } \\
\text { Balf.f.)Chamb.ex Cullen } \\
\text { et Chamb. }\end{array}$ & $\begin{array}{l}\text { Small evergreen } \\
\text { trees }\end{array}$ & Yanyuan County & $2900-4000 \mathrm{~m}$ & $\begin{array}{l}\text { Under } \\
\text { forests or } \\
\text { shrubs }\end{array}$ \\
\hline 55 & $\begin{array}{l}\text { Rhododendron } \\
\text { sparsifolia }\end{array}$ & $\begin{array}{c}\text { Rhododendron } \\
\text { hanceanum Hemsl. }\end{array}$ & Evergreen shrubs & Yanyuan County & $2400-2700 \mathrm{~m}$ & $\begin{array}{c}\text { Under } \\
\text { forests or } \\
\text { shrubs }\end{array}$ \\
\hline 56 & $\begin{array}{l}\text { Rhododendron } \\
\text { polytrichum }\end{array}$ & $\begin{array}{c}\text { Rhododendron } \\
\text { polycladum Franch. }\end{array}$ & $\begin{array}{l}\text { Evergreen erect } \\
\text { shrub }\end{array}$ & Yanyuan County & $3000-4300 \mathrm{~m}$ & $\begin{array}{l}\text { On the } \\
\text { meadow }\end{array}$ \\
\hline 57 & $\begin{array}{l}\text { Rhododendron } \\
\text { prairie }\end{array}$ & $\begin{array}{l}\text { Rhododendron } \\
\text { telmateium Balf.f. \& W. } \\
\text { W. Sm. }\end{array}$ & Undershrub & Yanyuan County & $3200-3800 \mathrm{~m}$ & $\begin{array}{l}\text { In the } \\
\text { thicket }\end{array}$ \\
\hline 58 & $\begin{array}{l}\text { Rhododendron } \\
\text { Zhaotong }\end{array}$ & $\begin{array}{l}\text { Rhododendron } \\
\text { tsaii Fang }\end{array}$ & Evergreen shrub & Yanyuan County & $2900-3380 \mathrm{~m}$ & $\begin{array}{l}\text { In the } \\
\text { thicket }\end{array}$ \\
\hline
\end{tabular}




\begin{tabular}{|c|c|c|c|c|c|c|}
\hline 59 & $\begin{array}{l}\text { Rhododendron } \\
\text { densiflora }\end{array}$ & $\begin{array}{c}\text { Rhododendron } \\
\text { fastigiatum Franch. }\end{array}$ & Evergreen shrubs & Yanyuan County & $3000-4500 \mathrm{~m}$ & $\begin{array}{l}\text { In the } \\
\text { thicket }\end{array}$ \\
\hline 60 & $\begin{array}{l}\text { Rhododendron } \\
\text { capillaris }\end{array}$ & $\begin{array}{l}\text { Rhododendron } \\
\text { trichostomum Franch. }\end{array}$ & Evergreen shrubs & Yanyuan County & $3000-4000 \mathrm{~m}$ & $\begin{array}{c}\text { Under } \\
\text { forests or } \\
\text { shrubs }\end{array}$ \\
\hline 61 & $\begin{array}{l}\text { Rhododendron } \\
\text { yunjinense }\end{array}$ & $\begin{array}{l}\text { Rhododendron } \\
\text { fortunei Lindl. }\end{array}$ & $\begin{array}{l}\text { Often Green } \\
\text { shrubs or small } \\
\text { trees }\end{array}$ & Yanyuan County & $1500-2000 \mathrm{~m}$ & $\begin{array}{l}\text { Woodlan } \\
\text { d retreat }\end{array}$ \\
\hline 62 & $\begin{array}{l}\text { Rhododendron } \\
\text { viridis }\end{array}$ & $\begin{array}{l}\text { Rhododendron } \\
\text { searsiae Rehd. et Wils. }\end{array}$ & Bush & Yanyuan County & $2300-3000 \mathrm{~m}$ & $\begin{array}{l}\text { Under } \\
\text { forests or } \\
\text { shrubs }\end{array}$ \\
\hline 63 & $\begin{array}{l}\text { rhododendron } \\
\text { bachii }\end{array}$ & $\begin{array}{c}\text { Rhododendron bachii H. } \\
\text { Lév. }\end{array}$ & Evergreen shrubs & Yanyuan County & $2400-2700 \mathrm{~m}$ & $\begin{array}{l}\text { In the } \\
\text { thicket }\end{array}$ \\
\hline 64 & $\begin{array}{l}\text { Rhododendron } \\
\text { brevipedunculata }\end{array}$ & $\begin{array}{c}\text { Rhododendron } \\
\text { brachypodum Fang et } P . \\
\text { S. Liu } \\
\end{array}$ & Bush & Yanyuan County & $1200-1500 \mathrm{~m}$ & $\begin{array}{l}\text { Woodlan } \\
\text { d retreat }\end{array}$ \\
\hline 65 & $\begin{array}{l}\text { Rhododendron } \\
\text { glandularis }\end{array}$ & $\begin{array}{c}\text { Rhododendron } \\
\text { adenogynum Diels }\end{array}$ & Evergreen shrubs & Yanyuan County & $3200-4200 \mathrm{~m}$ & $\begin{array}{c}\text { Under } \\
\text { forests or } \\
\text { shrubs }\end{array}$ \\
\hline 66 & Luo Mao Dujuan & $\begin{array}{c}\text { Rhododendron } \\
\text { detonsum Balf.f. et } \\
\text { Forrest }\end{array}$ & Evergreen shrubs & Yanyuan County & $3000-3900 \mathrm{~m}$ & $\begin{array}{l}\text { Under } \\
\text { forests or } \\
\text { shrubs }\end{array}$ \\
\hline 67 & $\begin{array}{l}\text { Rhododendron } \\
\text { splendens }\end{array}$ & $\begin{array}{l}\text { Rhododendron } \\
\text { heliolepis Franch. }\end{array}$ & $\begin{array}{l}\text { Often Green } \\
\text { shrubs or small } \\
\text { trees }\end{array}$ & Huili city & $3200-3700 \mathrm{~m}$ & $\begin{array}{c}\text { Under } \\
\text { forests or } \\
\text { shrubs }\end{array}$ \\
\hline 68 & $\begin{array}{l}\text { Rhododendron } \\
\text { lactiflorum }\end{array}$ & $\begin{array}{l}\text { Rhododendron } \\
\text { lacteum Franch. }\end{array}$ & $\begin{array}{c}\text { Often Green } \\
\text { shrubs or small } \\
\text { trees } \\
\end{array}$ & Huili city & $3300-3700 \mathrm{~m}$ & $\begin{array}{c}\text { Under } \\
\text { forests or } \\
\text { shrubs } \\
\end{array}$ \\
\hline 69 & $\begin{array}{l}\text { Rhododendron } \\
\text { rusticus }\end{array}$ & $\begin{array}{l}\text { Rhododendron } \\
\text { bureavii Franch. }\end{array}$ & Evergreen shrubs & Huili city & $3000-3700 \mathrm{~m}$ & $\begin{array}{l}\text { In the } \\
\text { thicket }\end{array}$ \\
\hline 70 & $\begin{array}{l}\text { Rhododendron } \\
\text { mianningensis }\end{array}$ & $\begin{array}{l}\text { Rhododendron } \\
\text { simsii Planch. }\end{array}$ & Evergreen shrubs & Mianning County & $3550-3900 \mathrm{~m}$ & $\begin{array}{l}\text { In the } \\
\text { thicket }\end{array}$ \\
\hline
\end{tabular}

\subsection{Horizontal distribution}

According to the statistics of counties (cities), wild Rhododendron plants are widely distributed in all counties (cities) in Panxi area, and Yanyuan County, Xichang City and Huili County have concentrated resource distribution. Among them, Yanyuan County has the most concentrated distribution, with 40 species, 3 varieties and 1 subspecies, accounting for $62.5 \%, 60 \%$ and $100 \%$ of the distributed species in Panxi area respectively; Secondly, there are 27 species, 2 varieties and 1 subspecies distributed in Xichang City, accounting for $42.2 \%, 40 \%$ and $100 \%$ of the distributed species in Panxi area respectively; The third is Huili County, which has 24 species, 1 variety and 1 subspecies, accounting for $37.5 \%, 20 \%$ and $100 \%$ of the distributed species in Panxi area respectively. There are 6 species and 1 subspecies and 5 species and 1 subspecies in Yuexi County and Xide County, respectively. See fig. 1 for species distribution of wild rhododendrons in counties (cities) of Panxi region. 


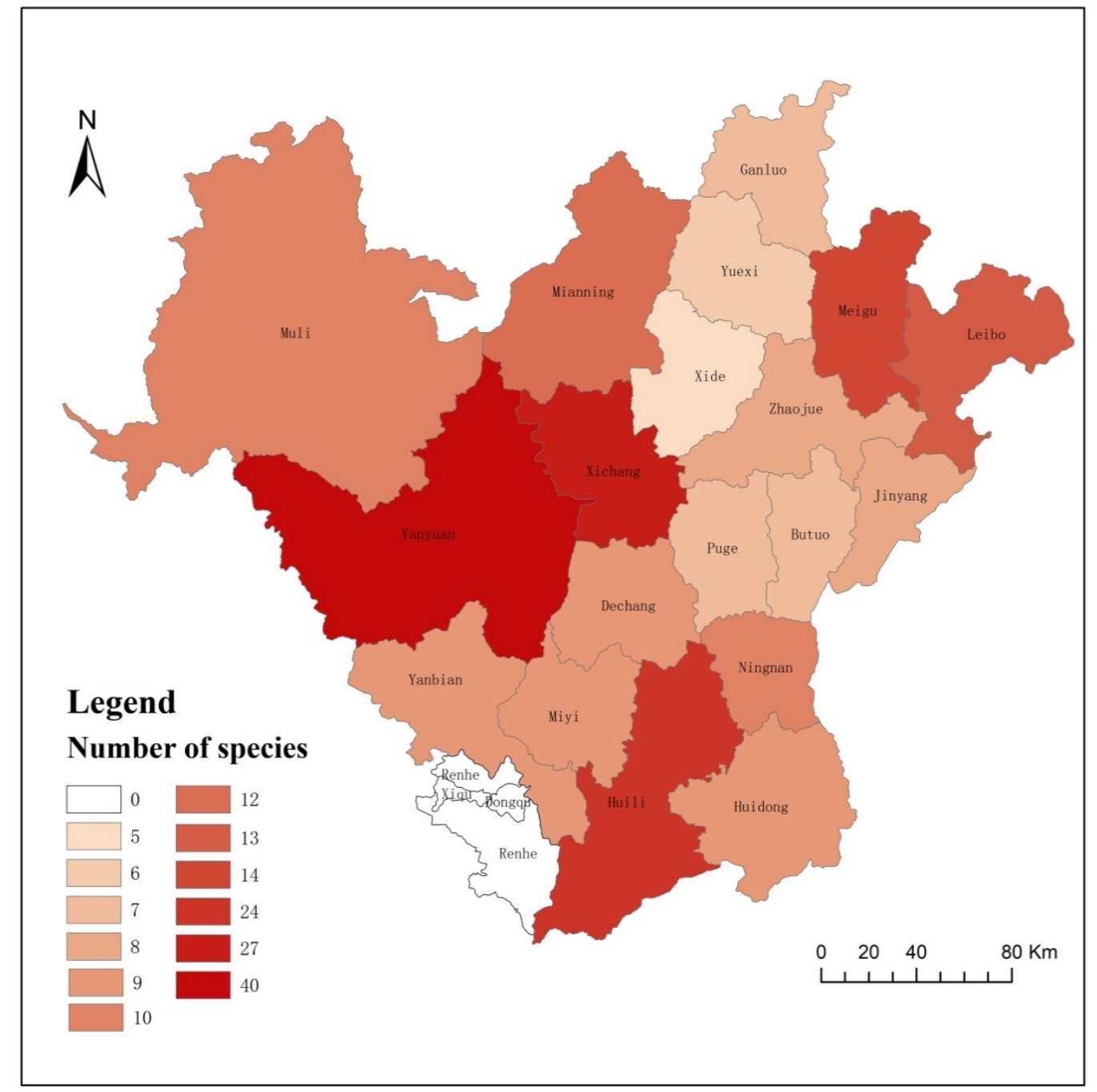

Figure 1. Schematic diagram of species distribution of wild Rhododendron in counties (cities) of Panxi area

\subsection{Vertical distribution}

According to the vertical distribution statistics, wild Rhododendron plants in Panxi area are distributed in the range of 1000-4500 $\mathrm{m}$ above sea level, and the altitude difference reaches $3300 \mathrm{~m}$. The vertical distribution of each species is quite different, and the species richness generally shows a trend of increasing first and then decreasing with the altitude. $2200-4000 \mathrm{~m}$ above sea level is the vertical section where the species of this genus are concentrated, with the highest species richness, including 60 species, 4 varieties and 1 subspecies. From the altitude distribution range of each species, the distribution range of Rhododendron cosmetology is the largest, ranging from 1300 to $4000 \mathrm{~m}$, with a vertical height difference of 2700 $\mathrm{m}$; Secondly, Rhododendron axillaris distributed in the range of 1500-3800 $\mathrm{m}$, with a vertical height difference of $2300 \mathrm{~m}$; The distribution range of Rhododendron Miyi is relatively narrow, and it is only found at $1700 \mathrm{~m}$ above sea level at present. The altitude distribution of wild Rhododendron species in Panxi area is shown in Figure 2. 


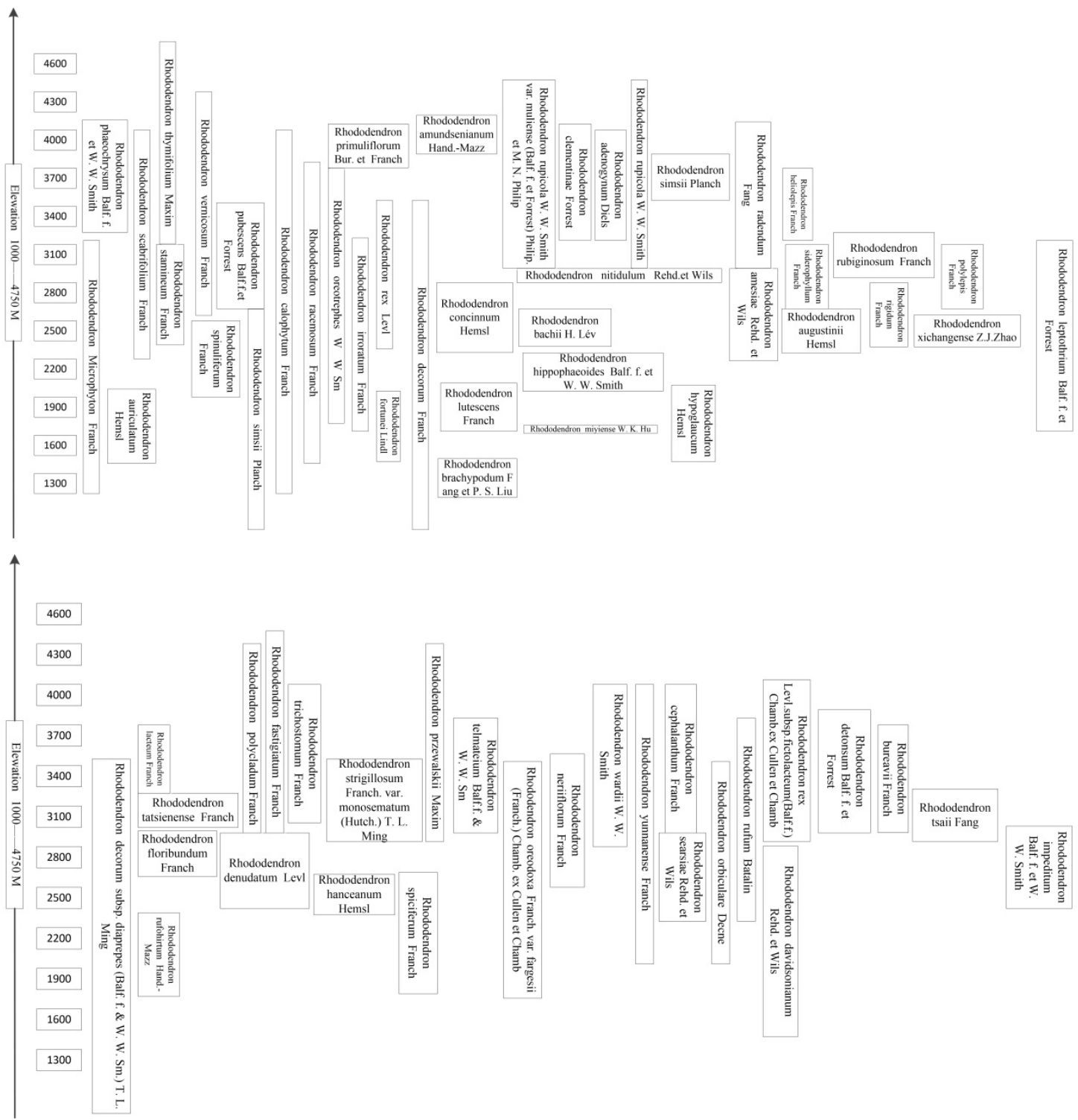

Figure 2. Schematic diagram of altitude distribution of wild Rhododendron plants in Panxi area

\subsection{Habitat distribution}

According to the statistics of vegetation types, there are 19 species and 1 variety of wild Rhododendron plants distributed under forests in Panxi area, accounting for 29.7\% and $20 \%$ of the total distribution species; There are 18 species and 2 varieties distributed in shrubs, accounting for $28.1 \%$ and $40 \%$ of the total distribution species; There are 22 species, 2 varieties and 1 subspecies distributed under forests or shrubs, accounting for $34.4 \%, 40 \%$ and $100 \%$ of the total distribution species; There are 5 species distributed in meadow, accounting for $7.8 \%$ of the total distribution species. The habitat distribution of wild Rhododendron species in Panxi area is shown in Table 2.

Table2. Habitat distribution of wild Rhododendron species in Panxi area

\begin{tabular}{cccccccc}
\hline \multirow{2}{*}{$\begin{array}{c}\text { Serial } \\
\text { Number }\end{array}$} & Habitat Type & Grow & $\begin{array}{c}\text { Proportion } \\
(\%)\end{array}$ & Mutation & $\begin{array}{c}\text { Proportion } \\
(\%)\end{array}$ & Subspecies & $\begin{array}{c}\text { Proportion } \\
(\%)\end{array}$ \\
\cline { 3 - 7 } & Woodland retreat & 19 & 29.7 & 1 & 20 & 0 & 0 \\
1 & In the thicket & 18 & 28.1 & 2 & 40 & 0 & 0 \\
3 & $\begin{array}{c}\text { Under forests or } \\
\text { shrubs }\end{array}$ & 22 & 34.4 & 2 & 40 & 1 & 100 \\
4 & On the meadow & 5 & 7.8 & 0 & 0 & 0 & 0 \\
\hline
\end{tabular}




\subsection{Geographical spatial distribution and traffic accessibility}

According to the geographical spatial distribution of wild Rhododendron plants, they are distributed in all counties and cities in Panxi area except the eastern, western and Renhe districts of Panzhihua. Among them, the number of wild Rhododendron species in the north of Panxi area is small, and it is found in the field investigation that most of them are scattered and less concentrated. There are abundant species of wild Rhododendron in the eastern, western and southern regions, especially in Yanyuan and Xichang in the west and midwest, Huili in the south, and Meigu and Leibo counties (cities) in the east. In the field investigation, it is found that the concentrated contiguous distribution is very obvious, which is highly ornamental in full bloom and has good tourism development value. From the correlation with the existing scenic spots, at present, the most famous scenic spots in Panxi area are Lugu Lake Scenic Area and Qionghai-Lushan Scenic Area. Yanyuan
County, where Lugu Lake Scenic Area is located, and Xichang City, where Qionghai-Lushan Scenic Area is located, both belong to the areas with the largest number of species and the most obvious concentrated contiguous distribution, which lay a good foundation for the development of tourism resources. From the point of view of geographical spatial distribution and traffic accessibility, because wild azaleas are distributed in the fractured terrain areas with high altitude, complex and varied topography and large fluctuation, subways and expressways will deliberately avoid the fractured terrain areas due to construction requirements, so neither railways nor expressways directly reach the wild azaleas landscape areas with good tourism development value. Xichang City, which has the best traffic conditions, has a small economic service radius, and its radiation driving ability can only radiate to two rhododendron landscape areas, namely Zhaojue Qiliba and Puge Haikou Ranch, which attract the most tourists at present. Therefore, traffic accessibility is a major constraint factor in the development of tourism resources. See fig. 3 for the geographical spatial distribution of wild rhododendrons in Panxi area.

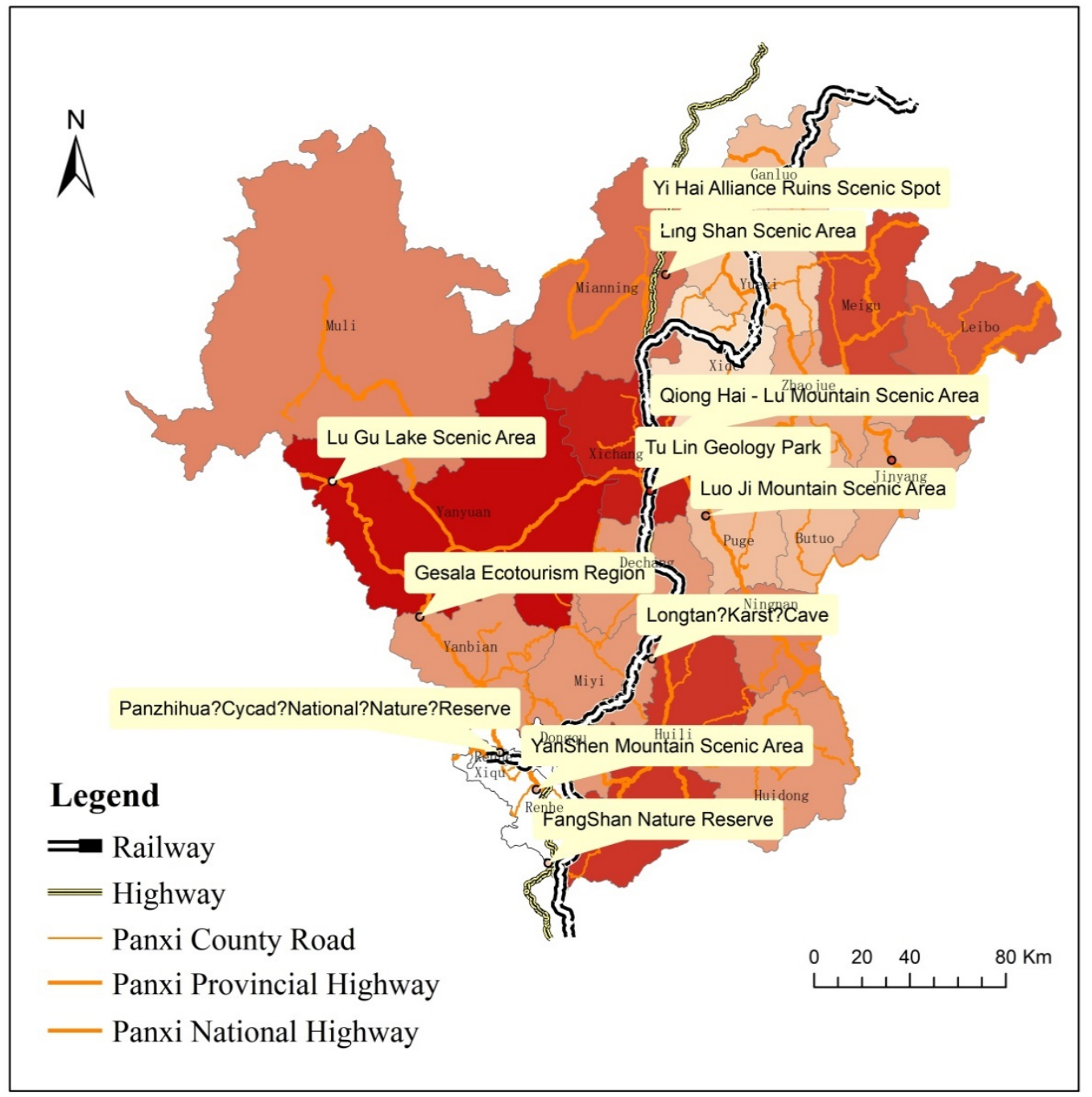

Figure 3. Schematic diagram of geographical spatial distribution of wild Rhododendron in Panxi area 


\section{Discussion and conclusion}

Panxi region has a special climate type, complex terrain and high altitude difference. Wild Rhododendron plants have a large number of species, wide distribution range, obvious concentrated distribution and long flowering period, forming an ornamental area with unique ornamental value. At present, the natural landscape of wild azaleas in Longelbow Mountain in Huili County, Houlongshan and Huangcao in Yanyuan County, Haikou Ranch in Puge County, Gukede and Riha in Zhaojue County, Yingpan Mountain and Aunt Mountain in Dalucao Township in Dechang County, Baicaopo in Jinyang County, Xiaoxiangling Ridge in Xile County, and Chajin in mana in Muli County have had great influence in Panxi area, and also have a certain popularity in Sichuan Province. With the unique beauty of azaleas, Panxi area, especially Liangshan Prefecture, is the key area of poverty alleviation in China, and it is the most important thing at present to consolidate and expand the achievements of poverty alleviation and effectively link up with rural revitalization. Industrial prosperity is the key to rural revitalization. One of the effective ways to achieve industrial prosperity in Panxi area is to develop rural tourism by using rich wild azalea resources in combination with local natural landscape and ethnic customs, cultivate new rural formats and models, activate the endogenous driving force of rural development, and promote the "trinity" development of rural production, life and ecology.

However, on the one hand, to develop rural tourism, the first priority is to attract tourists. Therefore, traffic accessibility is very important for the development and utilization of wild azalea tourism resources. At present, the eight azalea natural landscape groups with good development value mentioned above are only accessible by national or provincial roads, and it needs a long distance of township roads to reach the core landscape area. Therefore, in order to realize the economic development and utilization of wild rhododendron resources in Panxi area, it is necessary to further transform the traffic environment in Panxi area, strengthen infrastructure construction and improve traffic accessibility.

On the other hand, the flowering period of wild Rhododendron is mainly from May to July, and the ornamental value is poor outside the flowering period. Therefore, it is very important to use the existing traffic and scenic spots to establish landscape belts and extend the tourism value chain. In tourism development, other natural and cultural scenic spots around scenic spots can be integrated to create a landscape group integrating ethnic minorities' cultural customs and natural landscapes, enhance the agglomeration of regional tourism elements, and realize the effective development and utilization of tourism resources featuring wild Rhododendron plant resources in Panxi Plateau. Qionghai-Lushan Scenic Area is one of the most well-known scenic spots in Liangshan Prefecture. Besides, as it is located in the suburb of Xichang City. The regional economic radiation radius is stronger than other scenic spots. The Luoji Mountain
Scenic Area and Huanglian Soil Forest Scenic Area around the scenic spot can integrate Zhaojue Qiliba Rhododendron Landscape with Puge Haikou Ranch Rhododendron Landscape, enhance the gathering of regional tourism elements, create natural landscape groups, enhance the visibility of the landscape and attract more adult traffic; Lugu Lake Scenic Area can integrate Houlongshan and Huangcao Rhododendron landscape in Yanyuan County and Chajin Rhododendron landscape in mana, Muli County, and establish Lugu LakeHoulongshan in Yanyuan County and Huangcao-mana Chajin landscape belt in Muli County, so as to integrate Rhododendron viewing, Lugu Lake natural scenery and Yi-Tibetan ethnic customs experience, improve tourism quality and extend industrial chain. At the same time, special attention should be paid to the scarcity of wild Rhododendron resources, which cannot be recovered once destroyed. Therefore, the development of wild Rhododendron resources as tourism resources must be comprehensively utilized under the premise of protection.

\section{Project Fund:}

Key Project of Sichuan Education Department "Study on Conservation and Utilization of Wild Rhododendron Germplasm Resources in Panxi Area" (13ZA0269)

\section{About the author:}

Yuan Ying, female, associate professor, has been engaged in the research on plant classification and development and utilization of plant resources for a long time.

\section{References}

1. Huang Maoru . Rhododendron[M] . Shanghai: Shanghai Scientific \& Technical Publishers, 1999.

2. Wang Yuan, Ju Bo. Exploitation and utilization of Rhododendron in China $[\mathrm{J}]$. JOURNAL OF BIOLOGY, 2006, 23(1):43-44.

3. Wang Fuming, Zhou Jin. Influence of ecological factors on law of azalea's shape and growth on Luoji Mountain[J]. Journal of Southwest Minzu University (Natural Science Edition), 2003, 29 (6): 173-177.

4. Ding Bingyang, Jin Xiaofeng. Taxonomic Study on Rhododendron subgen Tsutsusi sensu Steumer(Ericaceae)[M'] . Beijing: Science Press, 2009, 246-255.

5. Zhang Xudong, Luo Qiang, Lin Jianlin. Rhododendrom resources suevey and developemnt of Panxi [J]. Forest By-Product and Speciality in China, 2007, 88(3):64-66.

6. Tian Qi, Ge Binjie, Wang Zhengwei. New Records of Rhododendron from Sichuan, China $[\mathrm{J}]$. Acta Botanica Boreali-Occidentalia Sinica, 2011, 31(1):192-194. 
7. Institute of Botany, the Chinese Academy of Sciences. Iconographia Cormophytorum Sinicorum [M]. Beijing: Science Press, 1974.

8. Yang Hanbi et al. Flora Reipublicae Popularis Sinicae (Tomus 57(1)) [M]. Beijing: Science Press, 1974:88.

9. $\mathrm{Li}$ Xiaofang, $\mathrm{Ma}$ Jinhua. Distribution \& Development and Utilization of Rhododendron Resources in Liangshan Prefecture of Sichuan[J]. Journal of Anhui Agricultural Sciences. 2009, 37 (27): 13060-13063. 